\title{
Clinical Trial Management Meeting Material
}

National Cancer Institute

\section{Source}

National Cancer Institute. Clinical Trial Management Meeting Material. NCI Thesaurus.

Code C115601.

A collection of agendas, presentation materials, and other documents generated for attendees during a clinical trial management meeting. 